\title{
DRESSEL 20 STAMPS FROM THE VERULAMIUM MUSEUM
}

\author{
Pedro Paulo A. Funari*
}

\begin{abstract}
FUNARI, P.P.A. Dressel 20 Stamps from the Verulamium Museum. Rev. do Museu de Arqueologia e Etnologia, São Paulo, 9: 143-161, 1999.
\end{abstract}

RESUMO: Este artigo trata dos selos de ânforas Dressel 20 custodiados no Museu de Verulamium (Reino Unido). Após uma introdução às ânforas Dressel 20 e à cidade romana de Verulamium (hoje Saint Albans, Reino Unido), há um catálogo de 39 selos diversos, calcados pelo autor. Cada selo é referido à literatura anterior, às prováveis olarias produtoras béticas e a selos datados contextualmente em outros lugares. Uma análise do tamanho dos selos e de sua cronologia é seguida de um estudo da origem das ânforas na Bética. $\mathrm{O}$ artigo conclui por enfatizar a importância de produzir catálogos de coleções de museus.

UNITERMOS: Ânforas romanas - Dressel 20 - Bretanha Romana - Olarias na Bética - Tamanho dos selos - Inscrições latinas.

\section{Introduction}

Dressel 20 amphorae are a very common find in Romano-British sites. These globular amphorae were used to carry olive oil from the Southern Spanish province of Baetica (Clak-Maxwell 1899; Bonsor 1931; Ponsich 1974, 1979). Its large body with handles and short neck ends in a basal knob. These amphorae, also known as Beltrán V. Ostia I and Callender II (Peacock \& Williams 1986: 136-140), are found in almost the whole Roman world. They were produced from the early Principate up to the mid third century AD (cf. map with potteries in Baetica). Dressel 20 amphorae usually bore inscriptions, tituli picti and stamps. Painted inscriptions are not easily found because of their difficult conservation in normal archaeological conditions. Stamps, on the other hand, are common-

(*) Departamento de História, Instituto de Filosofia e Ciências Humanas da Universidade Estadual de Campinas. ly found in British sites. Dressel 20 stamps found in Britain have been published since the Corpus Inscriptionum Latinarum, vol: VII, in the last century (inscriptions in the number 1331) and M. Callender (1965) was a pioneer in the publication of a comprehensive catalogue of stamps found up to the 1950s. In the last three deçades, there were a lot of stamps published in different papers, reports and books. However, there is still a lack of studies of all Dressel 20 stamps from specific sites. St. Albans is a case in point. Thanks to the Director of the Verulamium Museum, Dr. Chris Green, I was able to rub all the 49 Dressel 20 stored in that Museum and to study them in order to understand the consumption of Spanish olive oil in that Roman town.

Verulamium (St.Albans) was not an unimportant early Roman town: at the centre of the Catuvellauni territory, the Roman city developed with only peripheral military activity (Millett 1992: 77) and very soon it became a municipium ciuim romanorum (Rivet 1975: 111; cf. Reynolds 1975: 73; Tacitus, Ann., xiv,33). Verulamium was a large 


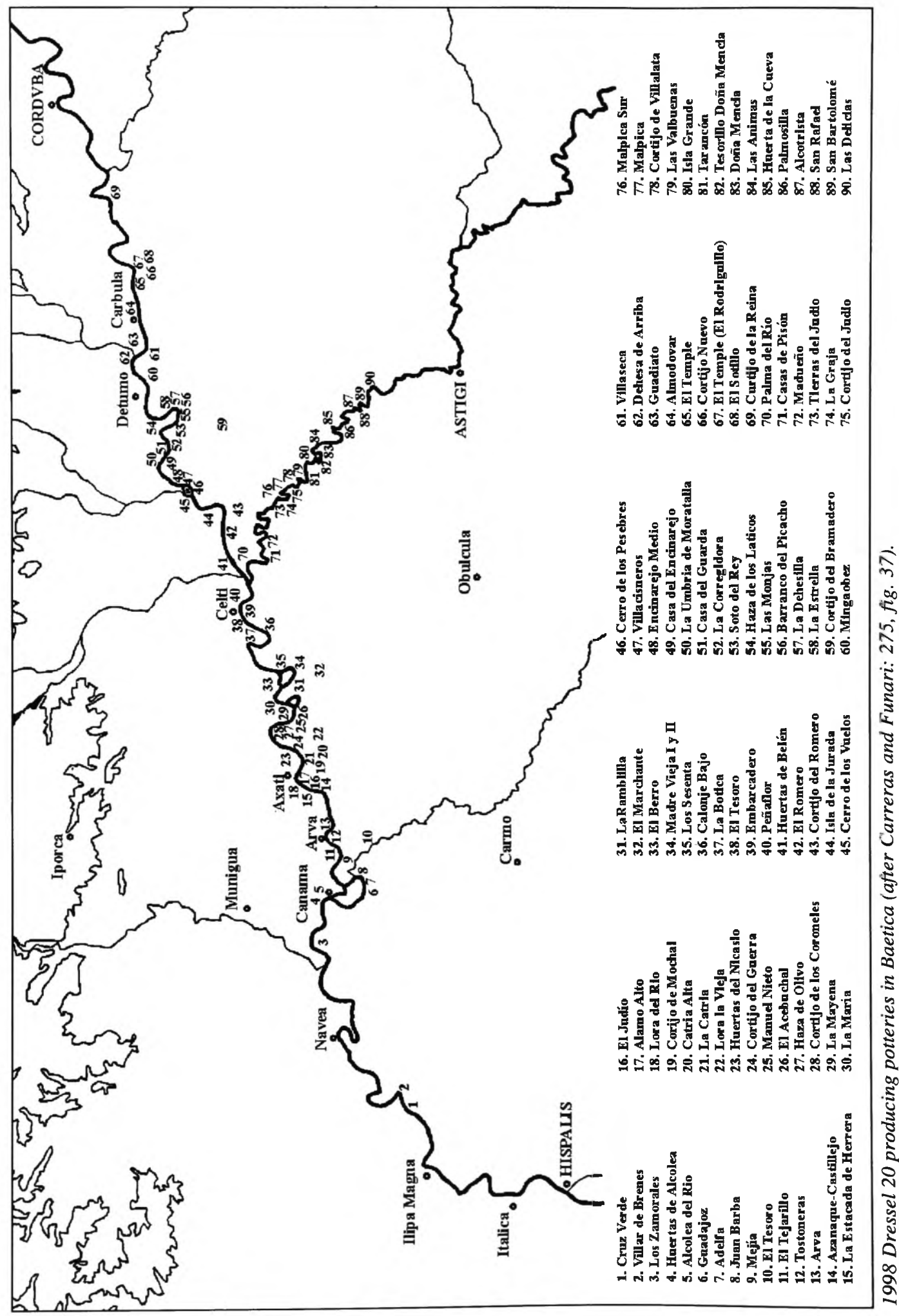


FUNARI, P.P.A. Dressel 20 Stamps from the Verulamium Museum. Rev. do Museu de Arqueologia e Etnologia, São Paulo, 9: 143-161, 1999.

settlement, comparing favourably with such continental sites as Avignon, Orange or Arles. The earliest town developed rapidly gaining a basilica and forum, a theatre, hypocaust, and walls (Collinwood \& Myres 1963: 189 et passim). Frere $(1972,1983)$ produced a comprehensive report of the archaeological work at Verulamium as well as an historical account of its development from its inception. Recent critical reappraisals of the evidence (Millett 1992: 69-71) do not change the fact that Verulamium was an important Roman town for the first two centuries of Roman rule in Britain. Verulamium town centre was vibrant and dynamic during the first and second centuries and the adornment of the city continued to the middle of the third century (Millett 1992: 107, 134), exactly the period when Dressel 20 were imported into the city. This explains the number of Dressel 20 stamps from the city (49) held at the Verulamium Museum, one of the largest collections from Britain. The recent publication of monographs on the overall consumption of olive oil in Britain (Funari 1996, Carreras \& Funari 1998) opens the way to the study of specific towns and the aim of this paper is thus to collect the stamps stored at a local museum, producing a catalogue of stamps, and to interpret the evidence available in the museum. Besides, the stamps will be studied as an odd evidence on the olive oil consumption at Verulamium both in terms of the importation from different potteries and producing areas in Baetica and in terms of consumption pattern changes in time.

\section{Catalogue of stamps}

The following abbreviations are used in the catalogue:

$\mathrm{FP}=$ Find Place.

$\mathrm{SP}=$ Storage Place.

$\mathrm{VM}=$ Verulamium Museum (the letters and numbers that follow the acronym refer to the data written on the sherd).

$\mathrm{CD}=$ Contextual date at Verulamium.

$\mathrm{OCD}=$ Other Contextual Dates (dates from other sites).

DOC: Dated by Other Criteria (typopological ones and others).

$\mathrm{CIL}=$ Corpus Inscriptionum Latinarum, $\mathrm{XV}, 2$. inches.
Testaccio A,B,C etc = location at the Testaccio, implying specific dates.

Bo. $=\mathrm{G}$. Bonsor, The archaeological expedition along the Guadalquivir, New York, 1931. The numbers refer to the catalogue of stamps.

Call. = M. Callender, Roman Amphorae. Oxford, 1965. The numbers refer to the catalogue of stamps.

Chic = G. Chic-Garcia, Epigrafía anfórica de la Bética. Seville, 1985. The numbers refer to the quoted page.

Kilcher = S.Martin-Kilcher, Die römischen Amphoren aus Augst und Kaiseraugst. Augst, 1987. The numbers refer to the catalogue of stamps.

Liou $=$ B. Liou, Inscriptions peintes sur amphores, Archaeonautica, 7: 55-141.

Ostia III = Clementina Panella, Appunti su un gruppo di anfore di prima, media e tarda età imperiale (secoli I-V d.C.), Studi Miscellanei, 21, Ostia III: 463-633.

Po. = M. Ponsich, Implantation rurale antique sur le Bas-Guadalquivir, vol.I, Madrid, 1974; vol. II, Paris, 1979. The numbers refer to volume, page and number in the catalogue of sites.

Port-Vendres II = D. Colls et al., L'épave PortVendres II et le commerce de la Bétique a l'époque de Claude. Paris, 1977.

Remesal $=\mathbf{J}$. Remesal, La annonna militaris y la exportación del aceite bético a Germania. Madrid, Universidad Complutense de Madrid, 1986. The numbers refer to the catalogue of stamps.

Rodriguez-Almeida, Bolli = E.

Rodriguez-Almeida, Bolli anforari di Monte Testaccio, Bolletino Communale, 1977: 199-247.

Verulamium I = Frere 1972.

Verulamium II = Frere 1983 .

Zevi = F. Zevi, Scavi ad Ostia, Notizie degli Scavi dell'Antichit... Romana, 26, 1972: 404-432.

Amphora production centres are located on the map.

The stamps are classified by the Roman nomen, usually the second letter of a set of three letters representing the Roman tria nomina. The names are considered to refer to estate owners or to owners of the olive oil inside the amphora. There are also the names of potteries, usually explicitly referred to as figlinae ("potteries"). After the name of the producing figlina there are also the names of the most probable regional centre in Baetica and finally the name of one of the three conuentus or "counties" in which the Baetis Valley was administratively divided (Hispalis = Seville; Astigi $=$ Écija; Corduba = Córdoba) . These data are studied in the latter part of this paper. 
FUN $\triangleleft$ 1. P.P.A. Dressel 20 Stamps from the Verulamium Museum. Rev. do Museu de Arqueologia e Etnologia, São Paulo. Y: 143-161, 1999.

\section{LANTSVRI}

\section{TESSVR}

Published in Verulamium III, xiv, no. 5 as L NI SVIR and Call. $901 \mathrm{~b}$

Cf. Chic 9.

FP: Verulamium.

$S P: V M(87.750)$.

Fabric: grey.

Handle: long.

Size: $1.8 \times 0.6$.

CD: AD 60-80.

Figlina: El Temple 59 (after Chic)

Almodóvar del Río

Corduba

Reading: LOANT()SVRI

\section{TATILIASI}

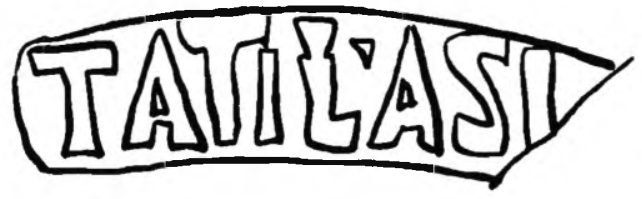

Unpublished.

Cf. Remesal 42, CIL 2717, Call. 1696, Po.1, 182, 191, nos. 122, 124, 136.

FP: Verulamium.

SP: VM (79.2591).

Fabric: grey.

Size: 2.6 x 0.8 .

OCD: Funari $41 \mathrm{a}=$ AD 160-300 (unpublished data).

Avenches = AD 50-110.

Kilcher 16 = AD 50-110.

AD 50-130.

DOC: AD 50-115 (Remesal, Will, Kilcher).

Figlinae: Adelfa 8, Juan Barva 9 \& Tostoneras 12

Arva

Hispalis

Reading: T()ATILI ASI<atici>
3. ...AC

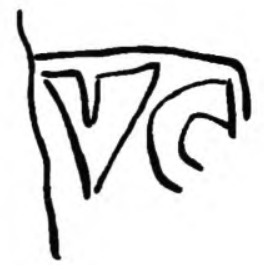

Unpublished.

FP: Verulamium.

SP: VM (82.155).

Fabric: grey.

Handle: long.

Reading: ...AC

4. ...C

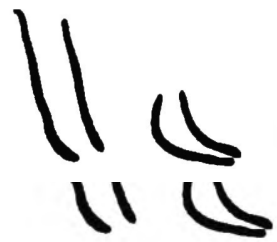

Unpublished.

FP: Verulamium.

SP: VM (VAKP 39 IA 82,149).

Fabric: reddish.

Handle: round.

a. C. ....

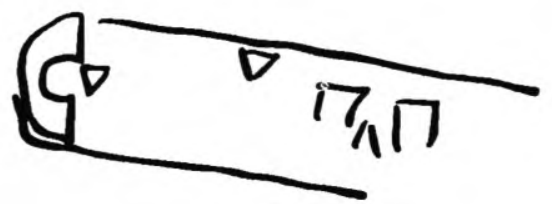

Unpublished.

FP: Verulamium.

SP: VM (site A, 1930, ins.I,bld.I, rammed filling north of cellar, 82.189).

Fabric: red.

Handle: medium.

Size: 0.7: 
5. Q.C.C

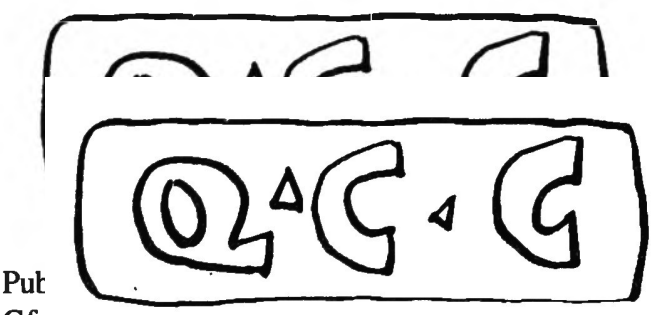

Cf.

Po.2,62,128.

FP: Verulamium.

SP (VM (87.1965).

Fabric: grey.

Size: 2.4 x 1.9 .

CD: AD 310-5 (= cellar filling)

OCD: Avenches = AD 90-120.

Augst $=$ AD 165-200.

Figlina: Las Sesenta 30

Mesa de Lora

Hispalis

Reading: Q()C()C()
6. ICI

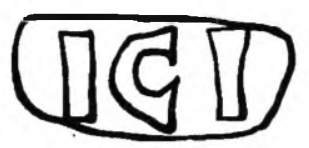

Published in Verulamium I, fig. 144, no. 2

Cf. Call. 826.

FP: Verulamium.

SP: VM (87.1968).

Fabric: grey-reddish.

Size: $1.3 \times 1.0$

CD: AD 130-150

Reading: $\mathrm{I}(\mathrm{C}() \mathrm{I}()$

\section{QCR}

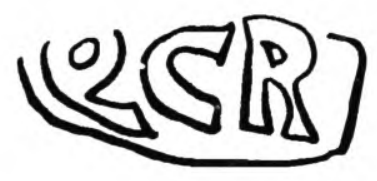

a. Q.C.C.I

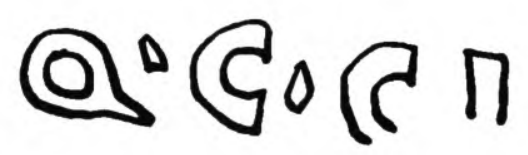

Unpublished.

FP: Verulamium.

SP: VM (87.1969)

Fabric: grey.

Handle: long.

Size: $2.6 \times 0.6$.

Reading: $\mathrm{Q}() \mathrm{C}() \mathrm{C}() \mathrm{I}()$
Unpublished.

Cf. Remesal 69, CIL 2763, Call. 1441.

FP: Verulamium.

SP: VM (82.180).

Fabric: grey.

Handle: long.

Size: $1.6 \times 0.7$.

OCD: Avenches = AD 40-90.

Kilcher $30=$ AD 30-70

AD 50-130 minimum: AD 70

AD 70-90

Funari 71a = AD 60-75 (unpublished data).

DOC: Trajanic (Rodríguez-Almeida, bolli, p. 236).

Reading: $\mathbf{Q}() \mathbf{C}() \mathbf{R}()$ 


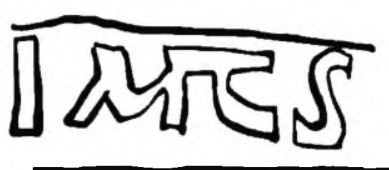

Unpublished.

Cf. CIL 2766, K,L. Call.1049.

Cf. Remesal 71, CIL 2998b, C,G (cf. CIL 4349),

Call. 1139f, Will 44, Po.1,145.

FP: Verulamium.

SP: VM (82.156).

Fabric: grey.

Handle: medium.

Size: 0.7 .

OCD: Testaccio, CIL 4349= AD 161 (M.MCSVR)

Figlina: Tejarillo 5 (if MMCSANTO)

Arva

Hispalis

Reading: $\mathrm{MM}$ ()C()S(), after Remesal or

M()MA()C()S(), after Dressel

\section{FIGED (retro)}

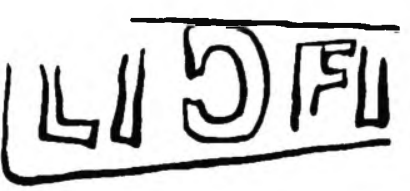

Unpublished.

Cf. Remesal 88 (FIGEDOPP), Po.2,165,38, CIL 2604, Call. 33.

FP: Verulamium.

SP VM (82.151).

Fabric: red.

Handle: round.

Size: 1.2 .

DOC: III c. (Remesal, typology).

Figlina: La Corrigidora 47

Posadas/Guadalcazar/Palma

Corduba

Reading: FIG(lina) ED(opinana)
Unpublished.

Cf. Call. 1447, Po.1,160,64.

FP: Verulamium.

SP: VM (87.1971).

Fabric: grey.

Handle: round-medium.

Size: $1.6 \times 0.9$.

Figlina: Arva 5

Arva

Hispalis

Reading: Q()F()AT()

\section{LFC ESCCVFP}

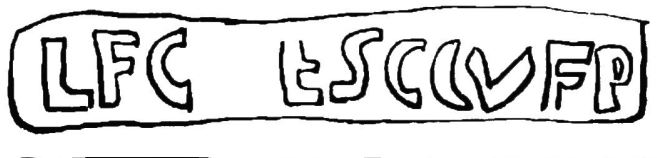

Unpublished.

Cf. Call. 851, CIL VII, 1331, 48, CIL 2587-94, Po.2,165,39 (=LFC and CVF).

FP: Verulamium.

SP: VM (82.185).

Size: $4.0 \times 0.7$.

OCD: CIL 3977-8 = AD 149.

Figlina: La Estrella 53

Palma del Río

Corduba?

Reading: L()F()C()CRESC(entis)CVF(iense) $\mathrm{P}($ ) or L()F()C()CRESC()C(larissimus)V(ir)FI(), after Remesal (1989). 
FUNARI, P.P.A. Dressel 20 Stamps from the Verulamium Museum. Rev: do Museu de Arqueologia e Emologia. São Paulo, 9: 143-161, 1999.

\section{a. ESCCVFP}

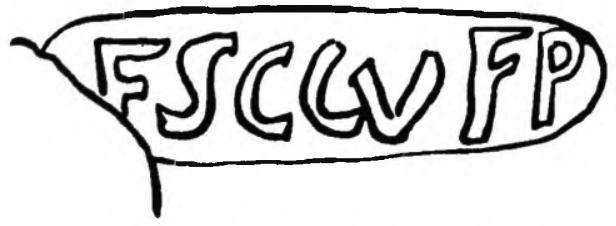

Unpublished.

FP: Verulamium.

SP: VM (82.187).

Fabric: grey.

Size: 2.6 x 0.7 .

Professor Remesal published in 1989 a study of these stamps, to which I send the reader to a complete analysis. It is based on the identification of a family $\mathrm{C}()$, being active for generations in the region. LFC would thus be identified as Fabius Cilo Septiminus Acilianus Lepidus Fulcianus, clarissimus uir, ordinary consul in AD 204, LFCCRESCCVF being another person of the same family (Remesal 1989: 143-5). Dressel found LFCCVFS in forma littera M (= AD 145-161) and LFCCVFS (retro) in forma littera L (= AD 179-180), earlier thus than the proposed identification with Lucius Fabius Cilo (Remesal 1989: 144). Anyway, if $\mathrm{C}() \mathrm{V}()$ refer to clarissimus uir then there is a need to explain CIL 2587, CVFIENSE.
Ostia = Flavian or earlier (cf. Zevi \& Pohl 1970: 46)

Figlina: Peñaflor 37

Peñaflor

Hispalis

Reading: $\mathrm{Q}() \mathrm{F}() \mathrm{C}()$

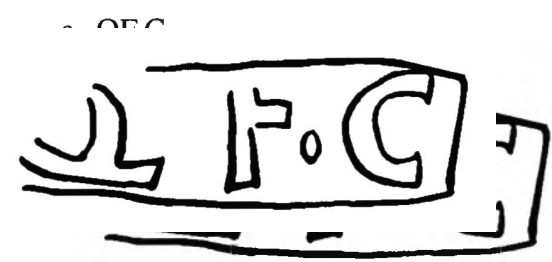

Published in Verulamium I. fig. 1H. n. 7.p. 370. FP: Verulamium. SP: VM (87.1967. Ins. XIV. III. 3b). Fabric: grey. Handle: long.

13. QFV (retro) or $\mathrm{QFA}<\mathrm{T}>$

12. Q.FC

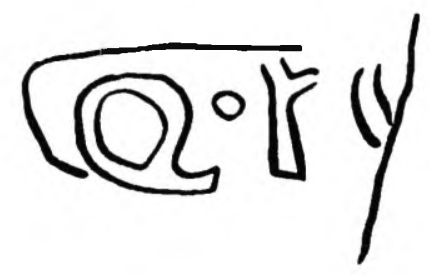

Unpublished.

Cf. Remesal 98 (QFC), CIL 2835. B.C.F.M. Call. 1449. Po.2.102,34. Liou (1987: 129-131).

FP: Verulamium.

SP: VM (87.1970, Ins.XIV. A. II. 27. 1958).

Fabric: grey.

Size: 0.7 .

OCD: Testaccio B.C.M = AD 1+5-161.

Testaccio $F=A D 161$.

Kilcher 50 = AD 90-130.

Liou $=$ AD 120-125.

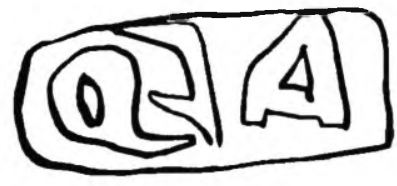

Published in Verulamium III. tig. 117.n.9 as QFATB (?).

Cf. CIL 2873 (QFV retro).

FP: Verulamium. SP: VM (87.753. Ins. XXVIII. bld.3. 58D XX+).

Fabric: reddish.

Handle: medium.

Size: $1.7 \times 0.7$.

CD: AD 1+0-160.

Figlina: if QFAT $=$ Ar7 a 5

Anvia

Hispalis

Reading: $\mathrm{Q}() \mathrm{F}() \mathrm{V}()$ or $\mathrm{Q}() \mathrm{F}() \mathrm{AT}()$ 
FUNARI, P.P.A. Dressel 20 Stamps from the Verulamium Museum. Rev. do Museu de Arqueologia e Etnologia, São Paulo, 9: 143-161, 1999.

14. D.I.A

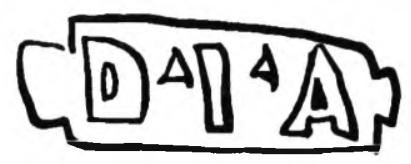

Unpublished.

Cf. Call. 535, CIL 2918a,B,C,D., Po.2,85,28; 91,57, Remesal 132.

FP: Verulamium.

SP: VM (82.181).

Size: $1.8 \times 0.6(1.4 \times 0.6)$.

OCD: Testaccio B,C = AD 145-161.

Figlinae: La María 32 and El Berro 33

Lora del Río

Hispalis

Reading: $\mathrm{D}(\mathbf{I}() \mathrm{A}()$

a. D.I.A (litteris cauis)

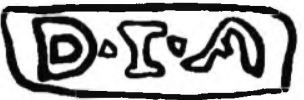

Published in Verulamium I, fig. 144, n.1, p. 370.

FP: Verulamium.

SP: VM (87.1964, T VI14, IIC, room 4).

Fabric: grey.

Size: $1.3 \times 0.4$.

CD: AD 140-150.

\section{II IVNI.MELISS}

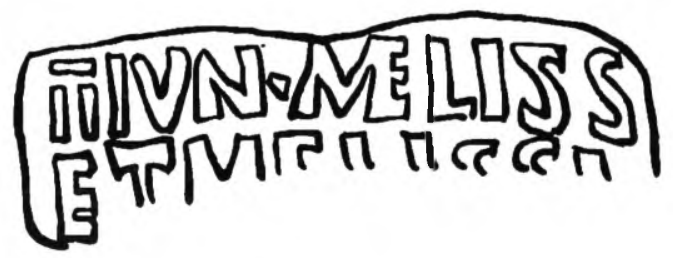

\section{ETMELISSE}

Unpublished?

Cf. Remesal 157, CIL 2967, Call. 878, Bo.80.

FP: Verulamium.

SP: VM (28.1012, GOR 80, 3917-2016).
Fabric: red.

Handle: round.

Size: $2.0 \times 0.9$.

OCD: Testaccio H = AD 214-222 (LIVNIMELISSI)

Kilcher $69=$ AD 210-250

Figlina: Las Delicias 71

Segovia

Astigi

Reading: II IVNI MELISSI ET MELISSE

a. II IVN ME

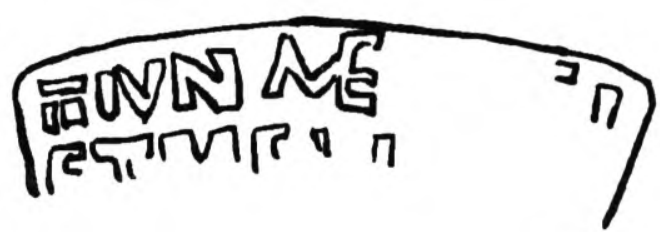

\section{ET MELI}

Unpublished.

FP: Verulamium.

SP: VM (82.186).

Fabric: red.

Handle: round.

Size: 2.11 x 0.8 .

16. L.I.T

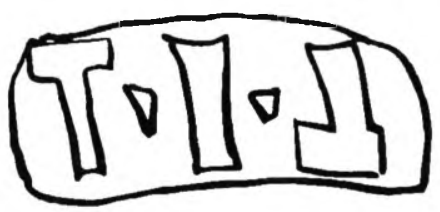

Unpublished.

Cf. Remesal 148, CIL 2947, G,K,L, Po.2,36,71 and 40,73 .

FP: Verulamium.

SP: VM (82.148.

Fabric: red.

Handle: round.

Size: 1.9 x 0.8 .

OCD: Testaccio G = AD 160 .

Testaccio K,L = AD 179-180.

Kilcher $68=$ AD 170-180. 
FUNARI, P.P.A. Dressel 20 Stamps from the Verulamium Museum. Rev. do Museu de Arqueologia e Etnologia, São Paulo, 9: 143-161, 1999.

(Minimum: AD 160-179).

Figlina: Alamo Alto 17 \& La Catria 19

Mesa de Lora

Hispalis

Reading: L()I()T()

\section{ALFM}

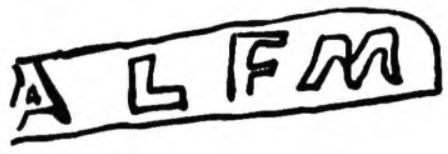

Unpublished, unparalleled.

FP: Verulamium.

SP: VM (82.182)

Fabric: grey.

Handle: long.

Size: $2.0 \times 0.5$.

Reading: $\mathrm{A}() \mathrm{L}() \mathrm{F}() \mathrm{M}()$

18

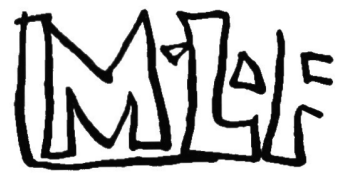

एᄂㅣㄴㄷㅏ

Unpublished; interpreted by Carreras (unpublished data) as an unparalleled stamp MLFN.

Cf. Call. 1130, CIL 2973, K, = MLFCP.

FP: Verulamium.

SP: VM (82.152).

Fabric: grey.

Handle: long.

Size: $2.3 \times 0.8$.

OCD: Testaccio K = AD 179-180.

Reading: $\mathrm{M}() \mathrm{L}() \mathrm{F}() \mathrm{C}() \mathrm{P}()$ or $\mathrm{T}()$ or $\mathrm{L}()$ (Callender's reading: $\mathrm{M}() \mathrm{L}() \mathrm{F}() \mathrm{CL}()$

\section{NQOCC}

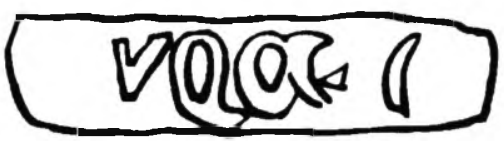

Unpublished.

Cf. If POROCODV, Remesal 191, CIL 2736, Call. 1370,18, Po.2,34,67; cf. CIL 3058 (PORODV).

FP: Verulamium.

SP: VM (82.1419, p.24-6a).

Fabric: grey.

Handle: long.

Size: $2.0 \times 0.6$.

OCD: Kilcher $92=$ AD 30-70.

AD 70-100. (Minimum: AD 70)

AD 30-90.

AD 20-90.

Nimega $=$ Flavian-Trajanic $(=$ Remesal 191).

Cologne $=$ Tiberian $(=$ Remesal 191$)$

Figlina: La Catria 19, Mochales 18, C.del Guerra 13, Olivo 24

Mesa de Lora

Hispalis

Reading: POR(tu)OC()ODV()?

Although the identification of this stamp is not sure, the OC linked letters suggest that it is a POROCODV or BROCODV stamp.

\section{L.M.A}

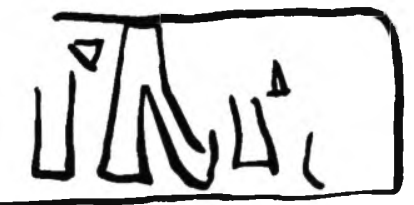

Published in Verulamium I, fig. 144, n.3, p.370 and quoted in Call. 887; unparalleled.

FP: Verulamium.

SP: VM (87.1966, Ins. XIV, a, III).

Size: $1.6 \times 0.10$.

Reading: $\mathrm{L}() \mathrm{M}() \mathrm{A}()$ 
FUNARI, P.P.A. Dressel 20 Stamps from the Verulamium Museum. Rev. do Museu de Arqueologia e Etnologia, São Paulo, 9: 143-161, 1999.

21. QMR

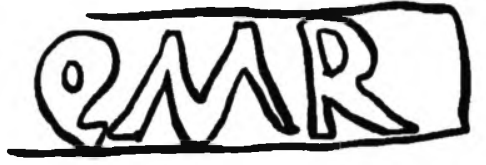

Unpublished.

Cf. Remesal 169h, CIL 3010c, C,F, Call.1481.

FP: Verulamium.

SP: VM (83.1446, UCP,b.vi,2s)

Fabric: red.

Handle: round.

Size: 0.7.

OCD: Kilcher $75=$ AD 70-210.

Testaccio C,F = AD 145-161.

Reading: Q()M()R()
23. APCO

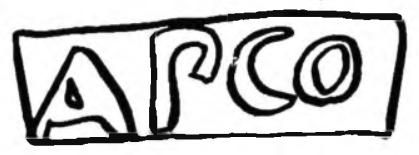

Published in Verulamium III, fig. 107, n.7.

Cf. CIL 3068, (APECO), Remesal 196 (APC), Call. 96.

FP: Verulamium.

SP: VM (87.755, Ins.XXVIII, 58 V 5).

Fabric: grey reddish.

Handle: long.

Size: 1.8 x 0.6 .

CD: AD 220-225.

OCD: in uentre elsewhere $=$ III century.

Figlina: La Catria 19

Mesa de Lora

Hispalis

Reading: $\mathrm{A}() \mathrm{P}() \mathrm{CO}()$ or $\mathrm{A}() \mathrm{P}() \mathrm{C}() \mathrm{O}()$

\section{DOMS}

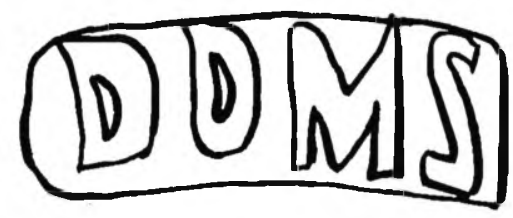

Published in Verulamium I, fig. 117, n.4.

Cf. Remesal 188, CIL 2800, B,C, Call. 552,

Po. 1,141,45.

FP: Verulamium.

SP: VM (87.748, building xxviii 2).

Fabric: grey.

Size: $2.0 \times 0.8$.

OCD: Testaccio B,C = AD 145-161.

CIL $3863=$ AD 146.

CIL $2800 \mathrm{~b}=$ AD 154.

Figlina: Alcolea 4

Alcolea

Hispalis

Reading: $\mathrm{D}() \mathrm{O}() \mathrm{M}() \mathrm{S}()$ (after Remesal) or DOM(iti)S (after Dressel).
24. AP.I or AP.M

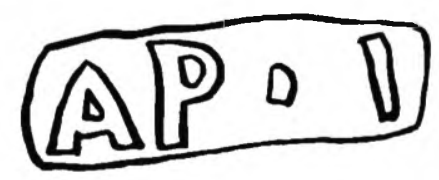

Published in Verulamium III, fig. 117, n.2 as AP.M. Cf. If APM, CLL 3072, Call. 105, Remesal 198.

FP: Verulamium.

SP: VM (87.756, Ins. XIV,57 V X 12).

Fabric: grey.

Size: $1.9 \times 0.6$.

CD: AD 130-145.

OCD: Avenches $=$ Trajanic.

Augst $=$ c. AD 50

Reading: $\mathrm{A}() \mathrm{P}() \mathrm{M}()$ or $\mathrm{I}()$ 
FUNARI, P.P.A. Dressel 20 Stamps from the Verulamium Museum. Rev. do Museu de Arqueologia e Etnologia, São Paulo, 9: 143-161, 1999.

25. L.Q.S

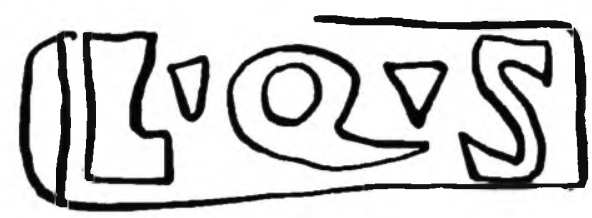

Unpublished.

Cf. Remesal 216, CIL 3109a, K,L, Call. 922, Po. $1,141,45 ; 187,125)$.

FP: Verulamium.

SP: VM (82.147, site A).

Fabric: grey-reddish.

Size: 2.4 x 1.9 .

OCD: Testaccio K, L = AD 179-180.

Kilcher $93=$ AD 150-250.

AD 130-190.

Cochester in uentre $=$ III century.

Figlinae: Alcolea 4 \& Tesoro 10

Canama Arva?

Hispalis

Reading: L()Q()S()

\section{GRADOS}

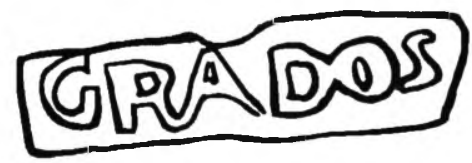

Unpublished.

Cf. Remesal 218, CIL 3121,H,L, Po.2,46,84, Rodríguez-Almeida, bolli, p. 236.

FP: Verulamium.

SP: VM (88.515, site A, 1930, ins.II, blg.I, room 2, tesselated floor).

Fabric: red.

Handle: round.

Size: $2.0 \times 0.5$.

OCD: Testaccio $H=A D$ 214-222.

Testaccio L = AD 179-180 (CIL 3121b, one line stamp).

Zevi $(1972: 417)=$ c. AD 250.

Figlina: Manuel Nieto 25

Mesa de Lora

Hispalis

Reading: $\mathrm{G}() \mathrm{R}() \mathrm{A}()<\mathrm{ET}>\mathrm{D}() \mathrm{O}() \mathrm{S}()$ (after Remesal)

or G()RA()DOS() (after Dressel).

\section{AXOFERRE}

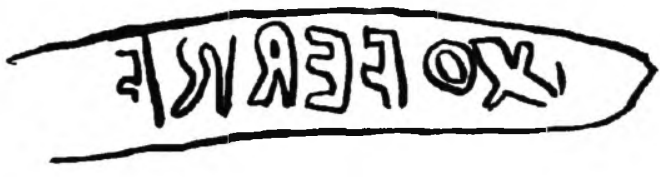

Unpublished.

Cf. If SAXOFERR, Remesal 249, CIL 3167g, B,C,F,M, Call. 1573, Po.2,111,117.

FP: Verulamium.

SP: VM (82.154).

Fabric: red.

Handle: medium.

Size: 0.7 .

OCD: CIL 4171 = AD 149 (=SAXOFERR).

Testaccio B,C,M = AD 145-161.

Testaccio B, C = AD 145-161 (SAXOFERR).

Figlina: Huertas del $R_{i} 038$

Palma del Río?

Corduba (cf. CIL 4171)

Reading: S>AXOFERR

This figlina saxoferreo is also quoted in a titulus delta inscription: $r$ cord cciilarca iiii figl (linae) Saxo<ferreo>/Orfito et Prisco (AD 149).

28. $\mathrm{MS}<\mathrm{P}>$

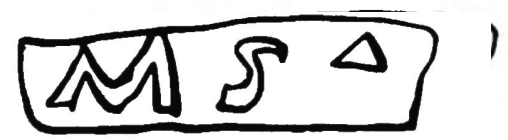

Unpublished.

Cf. CIL 3155, G,K,M, Call. 1180, Po.1,180,120,

Remesal 231.

FP: Verulamium.

SP: VM (82.183).

Fabric: grey.

Size: $1.9 \times 0.5$.

OCD: Testaccio G = AD 160 .

Testaccio $\mathrm{M}=145-161$.

Testaccio K = AD 179-180.

Figlina: Guadajoz 7

Arva

Hispalis

Reading: $\mathrm{M}() \mathrm{S}() \mathrm{P}()$ 
a. SA

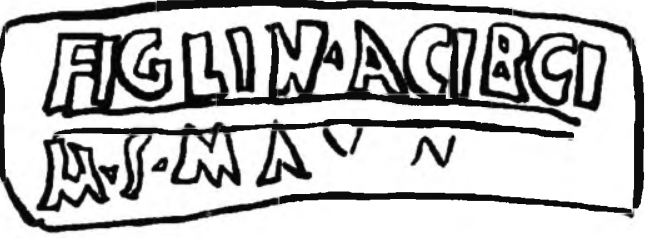

\section{M.S.MAV $<$ RIANI $>$}

Unpublished.

Cf. CIL XV,2, 2574a, Call. 18a; cf. Remesal 230 (MSM/ACIRGI).

Cf. Remesal 182 (MAVR), CIL 3027, Call.1034; Po.2,51,91, Remesal 44 (ACIRGIF), CIL 2575.

FP: Verulamium.

SP: VM.

Fabric: red.

Handle: round.

Sixe: $3.0 \times 1.0$.

DOC: III c.

Figlina : La Catria 19 ?

Mesa de Lora?

Hispalis?

Reading: FIGLINA ACIRGIENSIS / m()s() MAVRI (ani) or

$<$ ex> FIGLIN(is) ACIRG(ianis?) M()S()MAVRI... (after Dressel).

\section{SAE NB}

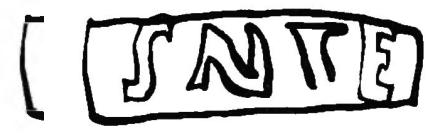

Published in Verulamium I, fig. 144, n.8 as SAENIN.B?

Cf. CIL 3304 (SAENIN.B), Call. 1559g; cf. CIL VII, $1331,118$.

FP: Verulamium.

SP: VM (80.2640, B I 68, pit7).

Fabric: grey.

Handle: long.

Size: 2.3 x 0.6 .

CD: AD 60-75.

Figlina: Huertas del Río 21

Lora del Río

Hispalis

Reading: SAENI N() B() or SAENIA()B()
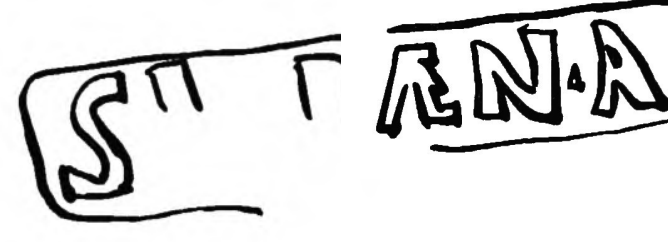

Unpublished.

FP: Verulamium.

SP: VM (88.523, bld.I,site A,1930, insula II).

Fabric: grey.

Handle: long.

Size: 1.7 x 0.7 .

Reading: SAENI

b. AEN.A

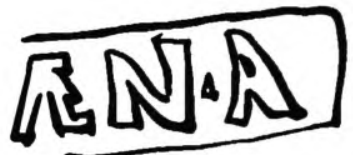

Published in Verulamium I, fig. $144, \mathrm{n} .9$ as SAENE or VENVS.

FP: Verulamium.

SP: VM (87.1963, B IV 9J, IID, room 43).

Handle: long.

Size: 0.6 .

CD: AD 150-155/160.

Reading: SAENIA

c. AEN.

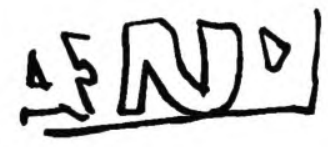

Unpublished.

FP: Verulamium.

SP: VM $(84.1449,1958$, ins.xiv).

Fabric: grey.

Handle: medium.

Size: 0.6.

Reading: SAENI 
31. SNTE

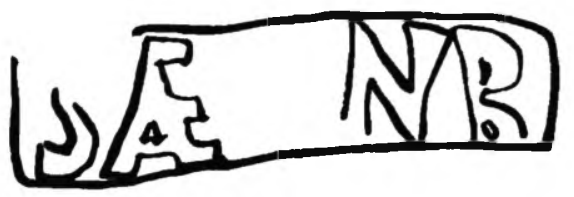

Published in Verulamium I, fig. 144, n.5 as PNSI or PNS; unparalleled.

FP: Verulamium.

SP: VM (87.1962, ins. XIV).

Fabric: reddish.

Handle: round.

Size: $1.6 \times 0.5$.

Reading: $\mathrm{S}() \mathrm{N}() \mathrm{T}() \mathrm{E}()$ ?

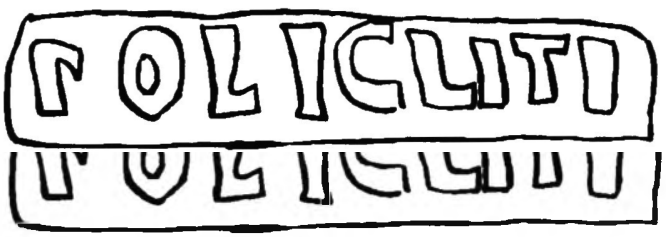

Unpublished.

Cf. Remesal 242, CIL 3176, Call. 1363.

FP: Verulamium.

SP: VM (82.179).

Fabric: grey.

Handle: long.

Size: $2.10 \times 0.6$.

OCD: Port-Vendres II $=$ c. AD 40.

Avenches $=$ AD 50-90.

Kilcher 112 = AD 30-50.

AD 50-110.

AD 0-50.

AD 30-70. Minimum: AD 50

AD 30-130.

AD 10-50.

Sheepen $=$ Pre-Boudiccan $=$ before AD 60.

Reading: C()SEMPRONI> POLYCLITI

32. Q.S.P

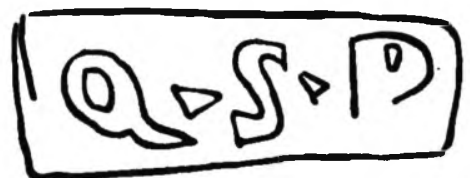

Unpublished.

Cf. Remesal 234, CIL 3156, Call. 1504.

FP: Verulamium.

SP: VM (82.153, b.III,23, central, 28.7.55,t.6).

Fabric: grey.

Handle: long.

Size: $2.0 \times 0.8$.

OCD: Vindolanda $=$ c. AD 90, before AD 105

(Funari 1991).

Avenches $=$ AD 90-110.

Ostia = c. 90 (= Ostia III, p. 124;254).

Kilcher $103=$ AD 90-110.

Figlina: Encinarejo Bajo 74

Posadas?

Corduba

Reading: $\mathrm{Q}() \mathrm{S}() \mathrm{P}()$

a. POLYCLITI

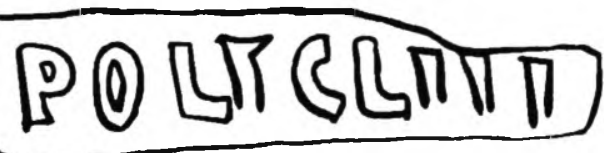

Published in Verulamium III, fig. $117, \mathrm{n} .8$ as POST.CVRTI.

FP: Verulamium.

SP: VM (87.749, Ver.1957, ins. XXVIII, b.l, y, II 23).

Fabric: grey.

Size: $1.8 \times 0.7$. 
34. OF

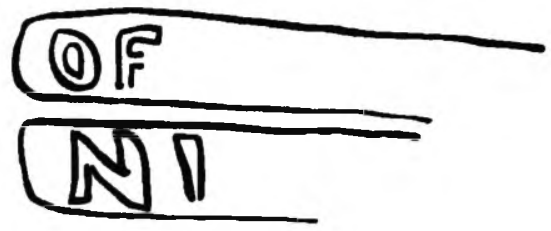

NI

Unpublished.

Cf.FSCIMINIANI, Remesal 251, CIL 3169a, I,H, Call. 1579, Bo.81-83.

FP: Verulamium.

SP: VM (82.1888)

Fabric: red.

Size: 0.10 .

OCD: Testaccio H,I = AD 214-222 (= SCIMINIANO).

Figlina: Las Delicias 71

Astigi

Astigi

Reading: OF(cinia) SCIMINIANO or O (leum?)

$F$ (figlina)

SCIMINIANO

\section{STATANIENSA}

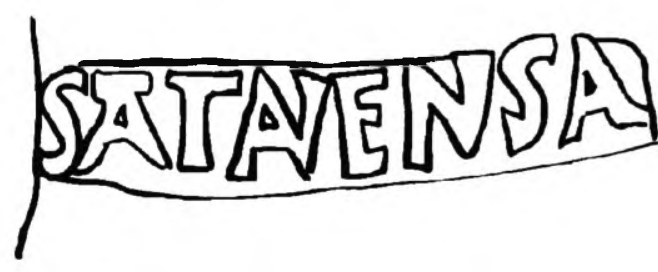

Published in Verulamium III, fig. 117, no.11, as STAT $<$ I $>$ AN $<$ I $>$ ENSA.

Cf. CIL 3192, Call. 1670b, Po.2,67,139, remesal 253.

FP: Verulamium.

SP: VM (87.752, ins. XVIII, 61 N).

Fabric: grey.

Size: 0.6.

OCD: Augst = AD 150-250.

Figlina: Tarancón 67

Segovia? Astigi?

Astigi

Reading: STATIANIENSIA
36. P.TM

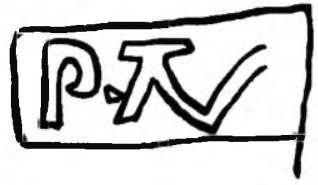

Published in Verulamium I, fig.144, n. 4, a P.M...; unparalleled.

FP: Verulamium.

SP: VM (87.1969, 37.e, Ver. 1958, ins. XIV, B.II, accumulation outside room 19 ).

Size: 0.6 .

Vessel size: $\mathrm{rim}=16.5 \mathrm{~cm}$.

neck $=10 \mathrm{~cm}$.

DOC: rim like Port-Vendres II vessels $=\mathrm{c}$. AD 40 .

Reading: $\mathrm{P}() \mathrm{T}() \mathrm{M}()$ ?

\section{VAL.FAVS}

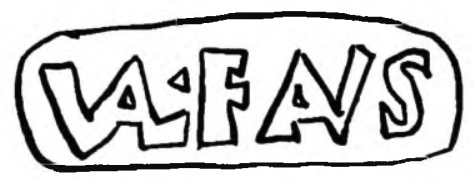

Published in Verulamium, fig. 117, n.12.

Cf. CIL 3217, Call. 1409d.

FP: Verulamium.

SP: VM (87.751, Ver.1957, ins. XVII, 57 K VII H 10).

Fabric: grey.

Handle: medium.

Size: $1.10 \times 0.7$.

Reading: VAL()FAVS() 
FUNARI, P.P.A. Dressel 20 Stamps from the Verulamium Museum. Rev. do Museu de Arqueologia e Etnologia, São Paulo, 9: 143-161, 1999.

\section{L.VARIONIS}

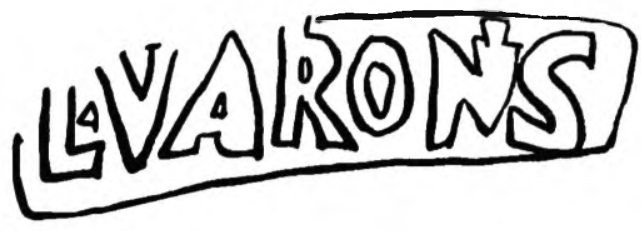

Published in Verulamium III, fig. 107, n. 6.

Cf. Call. 974.

FP: Verulamium.

SP: VM (87.754, ins. XXI, 59 L VI 5).

Fabric: grey.

Handle: medium.

Size: 2.6 x 0.6

OCD: Colchester $=$ before AD 65 .

Reading: L()VARIONIS

\section{V V}

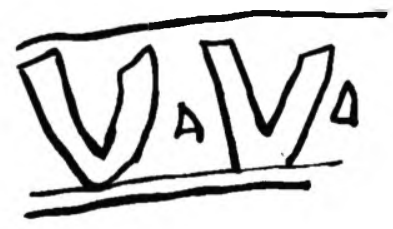

Unpublished.

Cf. CIL 3249 (V.V.I).

FP: Verulamium.

SP: VM (Mount Pleasant 1960 well).

Fabric: red.

Size: 0.9 .

Reading: $\mathbf{V}() \mathrm{V}()$

\section{Stamp size analysis}

Dressel 20 stamps usually follow some standard Roman inches sizes, most notably the half an inch or semuncia height $(\mathrm{c} .12 .5 \mathrm{~mm})$. More than thirty percent of all stamps from Verulamium follow this standard (Table 1) and it is probable that we should include the 0.7 stamps in this category, considering the lack of sharpness in both ours and ancient rulers. In that case, $58.6 \%$ of all stamps measure $c$. half a Roman inch, almost the same result for 489 Dressel 20 stamps found elsewhere in Britain $(57 \%$;

\begin{tabular}{c|c|c}
\multicolumn{3}{c}{ Table 1 } \\
\hline \multicolumn{3}{c}{ Stamp Size } \\
\hline \hline $\begin{array}{c}\text { Height in } \\
\text { Vnciae }\end{array}$ & $\begin{array}{c}\text { Number of } \\
\text { stamps }\end{array}$ & $\%$ \\
\hline 0.4 & 1 & $2.1 \%$ \\
0.5 & 4 & $8.6 \%$ \\
0.6 & 14 & $30.4 \%$ \\
0.7 & 13 & $28.2 \%$ \\
0.8 & 7 & $15.2 \%$ \\
0.9 & 3 & $6.5 \%$ \\
0.10 & 2 & $4.3 \%$ \\
1 & 2 & $4.3 \%$ \\
Total & 46 & $100 \%$ \\
\hline
\end{tabular}

unpublished data collected by the author). Many stamps follow specific standards, like sescuncia $x$ semuncia ( $=1.8 \times 0.6$; e.g. Stamp n.1), sescuncia $x$ digitus $(=1.6 \times 0.9$; e.g. Stamp n. 10$)$, sextans $x$ semuncia (= $2.0 \times 0.6$; e.g. Stamp n. 19), uncia et digitus $x$ semuncia $(=1.9 \times 0.6$; e.g. Stamp $n .24)$, quadrans $x$ uncia $(=3.0 \times 1.0$; e.g. Stamp n. 29), sextans et lycus $x$ semuncia $(=2.3 \times 0.6$; e.g. Stamp n. 30$)$ and sextans et semuncia $x$ semuncia $(=2.6$ x 0.6; e.g. Stamp n. 38; Table 2).

\section{Chronological analysis}

Thirty eight stamps from Verulamium are datable (Table 3). Antonine stamps are a plurality (42\%), with a good representation of late, Flavian and FlavianTrajanic samples. The small percentage of Pre-Flavian stamps remains to be explained, considering that at London, for example, $24 \%$ of all stamps are early, and at Verulamium only $7.8 \%$ (the figures for the third century are quite similar, $15.7 \%$ at Verulamium and $15 \%$ at London).

\section{Origin of the amphorae in Baetica}

Thirty stamps from Verulamium can be assigned to producing potteries in Baetica (Table 4 and 5). 
FUNARI, P.P.A. Dressel 20 Stamps from the Verulamium Museum. Rev. do Museu de Arqueologia e Etnologia, São Paulo, 9: 143-161, 1999.

Table 2

\begin{tabular}{|c|c|}
\hline \multicolumn{2}{|c|}{ Stamp Size, all stamps } \\
\hline $1-1.8 \times 0.6$ & $22-2.0 \times 0.8$ \\
\hline $2-2.6 \times 0.8$ & $23-1.8 \times 0.6$ \\
\hline $4 a-0.7$ & $24-1.9 \times 0.6$ \\
\hline $5-2.4 \times 1.9$ & $25-2.4 \times 1.9$ \\
\hline $5 a-2.6 \times 0.6$ & $26-2.0 \times 0.5$ \\
\hline $6-1.3 \times 1.0$ & 27- 0.7 \\
\hline $7-1.6 \times 0.7$ & $28-1.9 \times 0.5$ \\
\hline $9-0.7$ & $29-3.0 \times 1.0$ \\
\hline $10-1.6 \times 0.9$ & $30-2.3 \times 0.6$ \\
\hline $11-4.0 \times 0.7$ & $30 \mathrm{a}-1.7 \times 0.7$ \\
\hline $11 \mathrm{a}-2.6 \times 0.7$ & $30 b-0.6$ \\
\hline $12-0.7$ & $30 c-0.6$ \\
\hline $13-1.7 \times 0.7$ & $31-1.6 \times 0.5$ \\
\hline 14- $1.8 \times 0.6$ & $32-2.0 \times 0.8$ \\
\hline $14 a-1.3 \times 0.4$ & $33-2.10 \times 0.6$ \\
\hline $15-2.0 \times 0.9$ & $33 a-1.8 \times 0.7$ \\
\hline $15 a-2.11 \times 0.8$ & 34- 0.10 \\
\hline $16-1.9 \times 0.8$ & $35-0.6$ \\
\hline $17-2.0 \times 0.5$ & 36- 0.6 \\
\hline $18-2.3 \times 0.8$ & $37-1.10 \times 0.7$ \\
\hline $19-2.0 \times 0.6$ & $38-2.6 \times 0.6$ \\
\hline $20-1.6 \times 0.10$ & 39- 0.9 \\
\hline $21-0.7$ & \\
\hline
\end{tabular}

Comparing the percentages of stamps and of potteries located in each of the three conuentus exporting olive oil it is clear that the Hispalis region with only half the potteries was able to export two thirds of the stamped amphorae, while Astigi exported less than expected (13.3\% for $17.1 \%$ of all potteries) and Corduba even less (19.9\% for $30 . \%$ of all potteries). Comparing these data with those from London, it is possible to suppose
Table 3

\begin{tabular}{c|c|c|c|c|c|c}
\hline \multicolumn{5}{|c}{ Datable Stamps } \\
\hline \hline PR-FL & FL & FL-TR & HAD & C.150 & C. 180 & IIIC. \\
$3=$ & $6=$ & $6=$ & $1=$ & $13=$ & $3=$ & $6=$ \\
$7.8 \%$ & $15.7 \%$ & $15.7 \%$ & $2.6 \%$ & $34.2 \%$ & $7.8 \%$ & $15.7 \%$ \\
\hline
\end{tabular}

that Hispalis dominated both markets $(66.6 \%$ at Verulamium, $70 \%$ at London), Corduba was more successful at Verulamium (19.9\%) than at London (12.5\%) and Astigi more active at London (17\%) than at Verulamium (13.3\%). If we compare the data from Verulamium, London and six German sites (Nimega, Cologne, Mainz, Saalburg, Zugmantel, Nida) it is interesting to note that almost the same percentage come from La Catria and its region (Verulamium $=50 \%$; London $=53.6 \%$; Germania $=47 \%$ ), the single largest exporting area to the frontiers.

Considering the twenty eight stamps that can be dated and assigned to a producing pottery in Baetica , 21.3\% are Flavian and Flavian-Trajanic, $53.5 \%$ are Antonine and $24.9 \%$ are third century stamps. Amphorae from La Catria Region decline from $100 \%$ in Flavian times through $33.3 \%$ in the

\begin{tabular}{c|c|c|c|c}
\multicolumn{5}{c}{ Table 4 } \\
\hline \multicolumn{5}{c}{ Figlinae } \\
\hline \hline Conuentus & Stamps & $\%$ & Potteries & $\%$ \\
\hline Hispalis & 20 & 66.6 & 38 & 49.9 \\
$3-12$ & 1 & 3.3 & 2 & 2.6 \\
$13-33$ & 11 & 36.6 & 18 & 23.6 \\
$34-37$ & 2 & 6.6 & 4 & 5.2 \\
Astigi & 4 & 13.3 & 13 & 17.1 \\
$39 ; 62-71$ & 4 & 13.3 & 11 & 14.4 \\
Corduba & 6 & 19.9 & 25 & 32.8 \\
$38 ; 40-61$ & 5 & 16.6 & 23 & 30.2 \\
Encin. & 1 & 3.3 & 1 & 1.3 \\
Total & 30 & 100 & 76 & 100 \\
\hline
\end{tabular}


FUNARI, P.P.A. Dressel 20 Stamps from the Verulamium Museum. Rev. do Museu de Arqueologia e Etnologia, São Paulo, 9: 143-161, 1999.

Table 5

\begin{tabular}{l|c|c|c}
\hline \multicolumn{3}{|c}{ Stamps from Regions in Baetica } \\
\hline \hline & Verulamium & London & Germania \\
\hline Catria & $3=13.6 \%$ & $39=28.6 \%$ & $97=26 \%$ \\
$\begin{array}{l}\text { Catria } \\
\text { Region }\end{array}$ & $8=36.8 \%$ & $34=24.9 \%$ & $73=20 \%$ \\
Total & $11=50 \%$ & $73=53.6 \%$ & $170=47 \%$ \\
Arva & $1=4.5 \%$ & $9=6.6 \%$ & $19=5.6 \%$ \\
$\begin{array}{l}\text { Arva } \\
\text { Region }\end{array}$ & $4=18.1 \%$ & $16=11.7 \%$ & $43=11 \%$ \\
Canama & $2=9.0 \%$ & $16=11.7 \%$ & $44=12 \%$ \\
Delicias & $1=4.5 \%$ & $11=8 \%$ & $29=8 \%$ \\
$\begin{array}{l}\text { Delicias } \\
\text { Region }\end{array}$ & $3=13.6 \%$ & $0=0 \%$ & $2=0.5 \%$ \\
Total & $22=100 \%$ & $136=100 \%$ & $361=100 \%$ \\
\hline
\end{tabular}

Antonine period up to their absence in the third century. This is in contrast to the stable figures in London $(24.9 \% ; 27.7 \% ; 20 \% ; 22 \% ; 23.8 \%)$ but it is difficult to say if the smaller sample from Verulamium (only 28 stamps) in comparison with London (120 stamps) is not at least a partial explanation for this difference. On the other hand, the material from Corduba is more evenly represented at Verulamium $(50 \% ; 24.9 \% ; 28.5 \%)$ than at London $(3.5 \% ; 37.4 \% ; 5 \%)$.

\section{Conclusion}

The collection of Dressel 20 stamps from Verulamium adds to our knowledge of olive oil consumption in Roman Britain, particularly in the civilian area in the South and East of the province. The main conclusions are:

1. Hispalis producers exported to Verulamium at the average level of Britain (Verulamium $=66.6 \%$; Britain $=70.4 \%$ ), while Corduba exported much more to Verulamium than the average (Verulamium $=19.9 \%$; Britain $=6.5 \%$ ). In that case, the closest parallel is to be found in London. Astigi producers, as a consequence, exported less than the average (Verulamium $=13.3 \%$; Britain $=22.8 \%$ ) and the closest parallel is found at Caerleon $(13.6 \%)$;

2. In chronological terms, Pre-Flavian stamps are underrepresented at Verulamium $(7.8 \%$; Britain $=$ $39.5 \%$ ), as is the third century material (Verulamium $15.7 \%$; Britain $=20.9 \%$ ). As a consequence, Flavian and Flavian-Trajanic stamps represent $31.4 \%$ while for Britain the figure is only $12.7 \%$. Perhaps this could be explained by the development of the city at this period, although it could also be the result of different excavation data.

The detailed study of Dressel 20 stamps from different sites is the only way of enhancing our understanding of the olive oil consumption in Roman Britain (Funari 1991: 71). Moreover, the publication of stamps from museum collections is a way of producing catalogues to be used by other scholars interested in the stored material.

\section{Acknowledgements}

I am particularly indebted to Dr. Chris Green and to the Verulamium Museum for the access to the stamps. I owe thanks also to the following colleagues who forward papers (sometimes unpublished ones), exchanged ideas and helped me in different ways: César Carreras, José Remesal and Beth Richardson. 
FUNARI, P.P.A. Dressel 20 Stamps from the Verulamium Museum. Rev. do Museu de Arqueologia e Etnologia, São Paulo, 9: 143-161, 1999.

\title{
First letter index of stamps
}

\author{
AC (3) \\ AEN. (30C) \\ AEN.A (30B) \\ ALFM (17) \\ AP.I (24) \\ APCO (23) \\ AXOFERRE (27) \\ C (4) \\ D.I.A (14) \\ D.I.A (14A) \\ DOMS (22) \\ ESCCVFP (11A) \\ FIGED (9) \\ FIGLINA.ACIRGI MS MAV (29) \\ GRADOS (26) \\ ICI (6) \\ IIIVN.MEETMELI (15A) \\ IIIVNIMELISSETMELISSE (15) \\ L.I.T (16) \\ L.M.A (20) \\ L.Q.S (25) \\ L.VARIONIS (38) \\ LANTSVRI (1) \\ LFC ESCCUFP (11)
}

M.L.F (18)

MMCS (8)

$\mathrm{MS}<\mathrm{P}>(28)$

NQOCC (19)

OF NI (34)

P.TM (36)

POLYCLITI (33)

POLYCLITI (33A)

Q.C.C (5)

Q.C.C.I (5A)

Q.FC (12)

Q.SP (32)

QCR (7)

QF.C (12A)

QFAT (10)

QFV (13)

QMR (21)

SA (30A)

SAENB (30)

SNTE (31)

STATANIENSA (35)

TATILASI (2)

V.V (39).

VAL.FAVS (37)

FUNARI, P.P.A. Dressel 20 stamps from the Verulamium Museum. Rev. do Museu de Arqueologia

e Etnologia, São Paulo, 9: 143-161, 1999.

ABSTRACT: The paper deals with Dressel 20 amphora stamps stored at the Verulamium Museum (U.K.). After an introduction to the Dressel 20 amphorae and to the Roman city of Verulamium (nowadays Saint Albans, U.K.), there is a catalogue of 39 different stamps rubbed by the author. Each stamp is referred to earlier literature, to the probable producing potteries and to stamps dated contextually elsewhere. A stamp size and chronological analysis is followed by a study of the origin of the amphorae in Baetica. The paper concludes emphasizing the importance of producing catalogues from museum collections.

UNITERMS: Roman amphorae - Dressel 20 - Roman Britain - Potteries in Baetica - Stamp size - Latin inscriptions.

\section{References}

BONSOR, G.

1931 The archaeological expedition along the Guadalquivir (1889-1901). New York.

CALLENDER, $M$.

1965 Roman amphorae, with an index of stamps. Oxford: Oxford University Press.
CARRERAS, C.; FUNARI, P.P.A.

1998 Britannia y el Mediterraneo: Estudios sobre el abastecimiento de aceite bético y africano en Britannia. Barcelona: Universidad de Barcelona. CLARCK-MAXWELL, W.G.

1899 The Roman towns in the Valley of Baetis between 
FUNARI, P.P.A. Dressel 20 Stamps from the Verulamium Museum. Rev. do Museu de Arqueologia e Etnologia, São Paulo, 9: 143-161, 1999.

Cordoba and Seville. Antiquaries Journal, 56: 245-305.

\section{COLLINWOOD, R.G.; MYRES, J.N.L.}

1963 Roman Britain and the English Settlements. Oxford: Claredon Press.

FRERE, S.S.

1972 Verulamium Excavations, vol.I. The Society of Antiquaries, Oxford.

1983 Verulamium Excavations, vol.II. The Society of Antiquaries, Oxford.

FUNARI, P.P.A.

1991 Dressel 20 amphora inscriptions found at Vindolanda: the reading of the unpublished evidence. V.A. Maxfield; M.J. Dobson (Eds.) Roman Frontier Studies 1989. Exeter, University of Exeter Press: 65-72.

1996 Dressel 20 Inscriptions from Britain and the Consumption of Spanish Olive Oil. Oxford:

MILLETT, $M$ Tempus Reparatum.

1992 The Romanization of Roman Britain. Cambridge: Cambridge University Press.

PEACOCK, D.P.S.; WILLIAMS, D.F.
1986 Amphorae and the Roman Economy. An Introductory Guide. London: Longman.

PONSICH, M.

1974 Implantation rurale antique sur le Bas Guadalquivir, vol. I. Madrid: De Boccard.

1979 Implantation rurale antique sur le Bas-Guadalquivir, vol. II. De Boccard, Paris.

REMESAL, J.

1989 Tres nuevos centros productores de ánforas Dressel 20 y 23. Los sellos de Lucius Fabius Cilo. Ariadna, 6: 121-153.

\section{REYNOLDS, J.M.}

1975 Legal and constitutional problems. J.S. Wacher (Ed.) The Civitas Capitals of Roman Britain. Leicester, Leicester University Press: 70-75.

RIVET, A.L.F

1975 Summing up: some historical aspects of the $\mathrm{Ci}$ vitates of Roman Britain. J.S. Wacher (Ed.) The Civitas Capitals of Roman Britain. Leicester, Leicester University Press: 101-117.

ZEVI, F.; POHL, I.

1970 Ostia, saggi di scavo. Notizie degli Scavi, 7.24: 1. 